\title{
Influence of air humidity and contamination on electrical field of polymer insulator
}

\begin{abstract}
This paper presented the simulation results on electric field performances of medium voltage polymer type insulator while its mechanical conditions were reviewed. The purpose of this study is to investigate the electric field behaviour of $33 \mathrm{kV}$ polymer type insulator under different weather conditions by mean humidity and pollution. In addition, outdoor service environments of the insulator were reviewed as they are realized to have worldwide issues on degradation of polymeric material. Using 2D simulation, the electric field was found higher nearer to the end fittings whereby increased up to $38.89 \%$ under humidity and pollution. Also, the electric field behaviour along the cross section of weathersheds increased up to $661.59 \%$ under humidity and $547.30 \%$ under pollution. Meanwhile the weak points along the insulator were identified according to the magnitude of electric field strength. This research also verified the sharp edges and the thickness of the material of insulator as one of the factors that affect electric field strength.
\end{abstract}

Keyword: Composite insulator; Polymer; Suspension insulator; Electric field distribution 